\title{
Errata
}

Plane Deformations in Incompressible Nonlinear Elasticity

DEBRA POLIGNONE WARNE

Department of Mathematics, University of Tennessee, Knoxville, TN 37996, U.S.A.

\section{PAUL G. WARNE}

Division of Mathematics and Computer Science, Maryville College, Maryville, TN 37804, U.S.A.

Journal of Elasticity 52: 129-158, 1999.

The general equilibrium equations in terms of the components of the nominal stress tensor referred to cylindrical polar coordinates as given in $(2.14 \mathrm{a})-(2.14 \mathrm{c})$ on p. 139 of the above paper are free of any typographical errors; however, the expressions (A.20) and (A.21) of the Appendix, regrettably are not. We wish to point this out so that anyone using these equations does not unknowingly use equations containing misprints. To correct the first, the expression in brackets in (A.20) should be replaced by the left-hand side of the equation (2.14b) given in the paper; in particular, the term $\theta_{, Z} S_{Z \theta}$ in (A.20) should be replaced by $S_{Z \theta_{, Z}}$. To correct the second, the expression in brackets in (A.21) should be replaced by the left-hand side of the equation (2.14c) given in the paper; in particular there should be a + preceding the term $S_{Z z, Z}$. 